\title{
Response of Quartz Crystal Microbalance Loaded with Single-drop Liquid in Gas Phase
}

\author{
Li Yang $^{1,2, *}$ and Xianhe Huang ${ }^{1}$ \\ ${ }^{I}$ Frequency Control Laboratory, School of Automation \& Engineering, University of Electronic Science and Technology \\ of China, Chengdu, 610054, China \\ ${ }^{2}$ College of Electrical \& Information Engineering, Southwest University for Nationalities, Chengdu, 610041,China
}

\begin{abstract}
The frequency response of quartz crystal microbalance loaded by single-drop liquid is studied in this paper. Previous studies have shown that the relationship between resonant frequency and properties of liquid by completely immersing one side of the crystal in liquid. In this work, only localized portion of crystal was wetted by liquid droplet. Repeated experiment shows the relationship between liquid property include viscosity and density to resonant frequency. Furthermore, Theoretical formula describing the frequency change of the quartz crystal microbalance with liquid property is proposed. The predicted results showed distinct coincide with experimental results.
\end{abstract}

Keywords: Quartz crystal microbalance, single-drop, gas phase.

\section{INTRODUCTION}

The quartz crystal microbalance(QCM) has been widely used as highly sensitive sensor which commonly configure with electrode on both sides of a thin disk AT-cut quartz. The crystal can be electrically excited into resonance because of the piezoelectric properties. In the late 1950s, Sauerbrey found the relationship between resonant frequency and mass deposit on surface of quartz in gas phase [1],

$$
\Delta f=-\frac{2 f_{0}^{2}}{A\left(\mu_{q} \rho_{q}\right)^{\frac{1}{2}}} \Delta m
$$

Where $\Delta \mathrm{f}$ is the observed frequency change (in $\mathrm{Hz}$ ), $\mu_{\mathrm{q}}$ is the shear modulus, $\rho_{\mathrm{q}}$ is the density of the crystal, $\mathrm{A}$ is the active electrode area, and $\Delta \mathrm{m}$ is the mass change on the surface of the crystal (in ng). For the AT-cut quartz crystal $\left(\rho_{\mathrm{q}}=2.65 \mathrm{~g} / \mathrm{cm}^{3}, \mu_{\mathrm{q}}=2.95 \mathrm{~N} / \mathrm{m}^{2}\right)$. Since the work of Sauerbrey, the QCM has been widely used to monitor slight mass change(nanogram) in many research areas include biology, physics, medicine and so on. In the late 1980s, Kanazawa found the QCM oscillate in contact with liquid, the relationship between liquid property (such as viscosity, density) and resonant frequency was founded while immerse one surface of crystal in liquid [2]. The equation showed as follow:

$$
\Delta f=-f_{0}\left(\frac{\rho \eta}{\pi \mu_{q} \rho_{q}}\right)^{\frac{1}{2}}
$$

Where $\Delta \mathrm{f}$ is the difference in resonant frequency between the coated and bare crystal, $\eta$ is the viscosity of liquid and $\rho$ is the density of the liquid.it therefore extend the application of QCM to liquid media.
For Sauerbrey equation, the deposit thin film should be rigid thin and for Kanazawa equation, one surface of crystal should be immersed in liquid. if a microliter liquid dropped on the surface of QCM, the Sauerbrey equation and Kanazawa equation may not suitable for the interpretation of the frequency response of QCM. However, if the frequency response of QCM loaded by liquid droplet could be figured out, the condition that immerse one surface of crystal in liquid could be changed to drop one microliter liquid on surface of crystal, then, the application of QCM would be extended to more area and it must be more convenient.

In this paper, the frequency response of QCM loaded by liquid droplet was investigated. In particular, the resonant frequency shift was discussed in relation to the property of liquid such as viscosity and density. In addition, the resonant frequency of QCM loaded by microliter liquid has been measured. The results predicted by the theoretical model and obtained by the experiments are compared.

\section{PRINCIPLES OF QCM LOADED BY LIQUID DROP}

Liquid droplet resting on the surface of crystal takes the shape of a spherical cap, like (Fig. 1).

In which, $r_{d}$ is the base radius for liquid droplet, $r_{e}$ is the electrode radius; the $r_{d}$ smaller than $r_{e}$. Because it has small volume, the distort by gravity may negligent. Owing to the Newtonian properties of the liquid, the Liquid films in contact with the QCM do not behave as rigid layers and the films does not oscillate as a whole in sympathy with the crystal. With the drop of liquid, the frequency of the AT-cut crystal resonator would decreased and the frequency change can be described as follow [3]:

$\Delta \mathrm{f}=\frac{1}{\pi r_{d}^{2}} \int_{0}^{2 \pi} \int_{0}^{r_{d}} S(r, \theta) m(r, \theta) r d r d \theta$ 


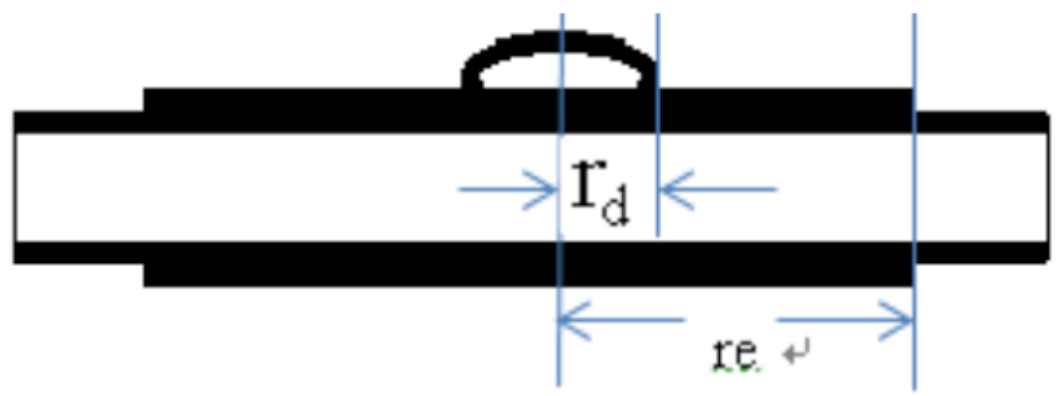

Fig. (1). Schematic view of single-drop liquid localized on the QCM surface.

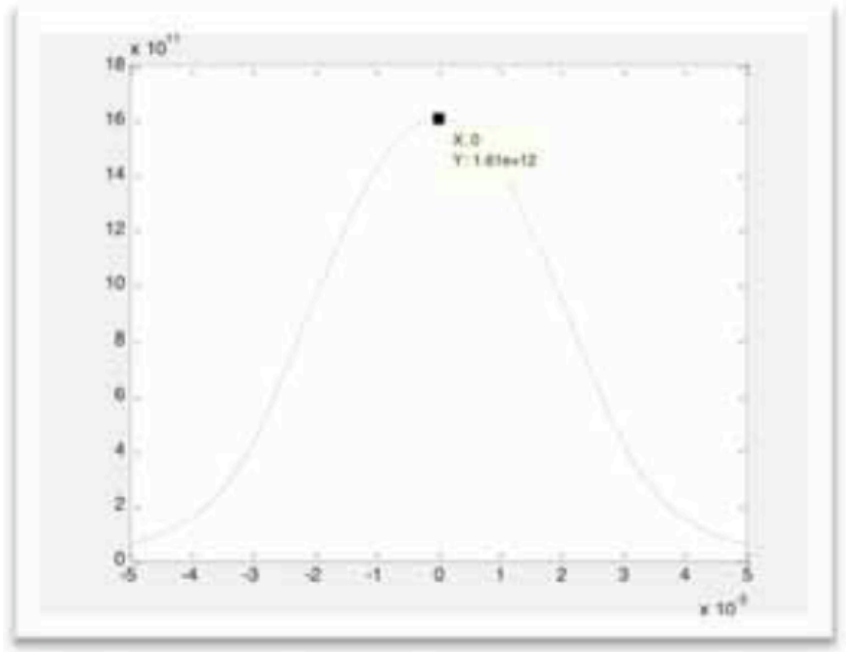

Fig. (2). The mass sensitivity along the radial direction.

In which, $S(r, \theta)$ is mass sensitivity function and represents the differential frequency change per mass change at a specific location, $\Delta \mathrm{m}(\mathrm{r}, \theta)$ is the effective added mass, $\mathrm{r}_{\mathrm{d}}$ is the radius for the localized or nonuniform mass deposit on the electrode, $r$ and $\theta$ are the polar coordinates of the point at which the mass is added. Mass sensitivity function could be calculated by equation (4):

$$
\mathrm{S}(\mathrm{r})=\frac{\left|u_{1}(r)\right|^{2}}{2 \pi \int_{0}^{\infty}\left|u_{1}(r)\right|^{2} r d r} C_{f}
$$

Where $u_{1}(r)$ is particle displacement amplitude , $C_{f}$ is sauerbery's sensitivity constant, $r$ is the distant from center. For particle displacement amplitude, the function could be written as equation [4] (5).

$u_{1}\left(x_{1}, x_{2}, x_{3}, t\right)=u_{1}\left(x_{1}, x_{3}\right) \sin \left(k_{2} x_{2}\right) \cos \omega t$

The surface plane of gold electrode was defined by $\left(\mathrm{x}_{1}, \mathrm{x}_{3}\right), k_{2}$ is the shear horizontal acoustic wavenumber in the $X_{2}$ direction. The equation (5) could be reduced as:

$\frac{c_{11}}{c_{66}} \frac{\partial^{2} u_{1}(r)}{\partial x_{1}^{2}}+\frac{c_{55}}{c_{66}} \frac{\partial^{2} u_{1}}{\partial x_{3}^{2}}+\frac{\omega^{2}-\omega_{i}^{2}}{c^{2}} u_{1}(r)=0$

Where $C_{i j}$ represent the elastic stiffness constant; $\omega_{i}=k_{2} \sqrt{\frac{c_{66}}{\rho}}$ is cutoff frequency, $c=\sqrt{\frac{c_{66}}{\rho}}$ is the acoustic wave velocity in crystal. Convert equation (6) into polar coordinate:

$r^{2} \frac{\partial^{2} u_{1}(r)}{\partial r^{2}}+\frac{\partial u_{1}(r)}{\partial r}+\frac{K_{I}^{2} r^{2}}{A} u_{1}(r)=0$

The equation (7) is a Bessel differential equation and the general solution could be shown as

$$
u_{1}(r)=\begin{aligned}
& C_{1} J_{0}\left(\frac{K_{I}}{\sqrt{A}} r\right)+C_{2} N_{0}\left(\frac{K_{I}}{\sqrt{A}} r\right) \\
& C_{1} I_{0}\left(\frac{K_{I}}{\sqrt{A}} r\right)+C_{2} K_{0}\left(\frac{K_{I}}{\sqrt{A}} r\right)
\end{aligned}
$$

Where $\mathrm{J}_{0}, \mathrm{Y}_{0}, \mathrm{I}_{0}$, and $\mathrm{K}_{0}$ are the Bessel's functions of the first and second kind with order zero and the modified Bessel's function of the first and second kind with order zero. The particle displacement amplitude $u_{1}(r)$ could be calculated by function (8), submitted solution into equation (4), the mass sensitivity functions(r) could be calculated. In this study, a 3rd overtone $10 \mathrm{MHz}$ AT-cut crystal was used. The electrode radius and thickness are set as $1000 \dot{A}$ and $3 \mathrm{~mm}$ respectively. Fig. (2) shows the variations of the differential mass sensitivity along the radial direction.

From Fig. (2) we can see that the mass sensitivity of the QCM is the largest in the center of electrode and decrease monotonically along the radial direct. Notably, the mass 


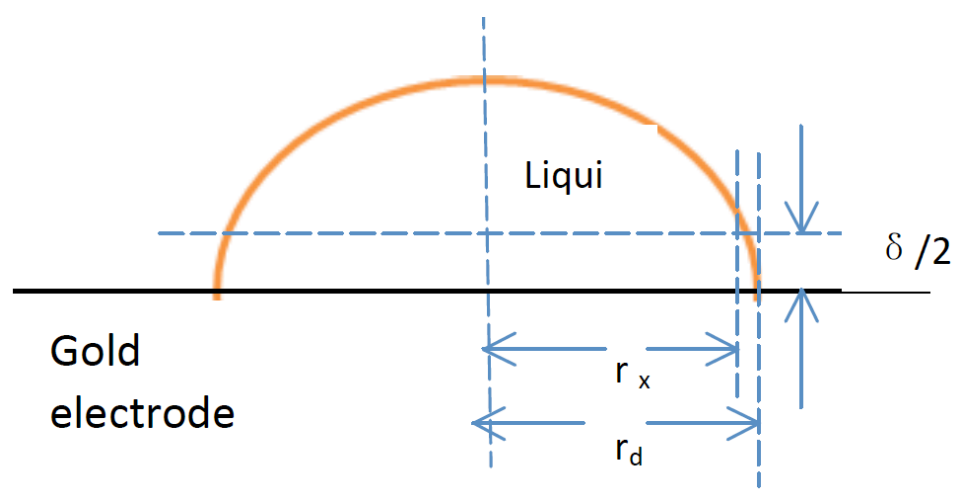

Fig. (3). View of single-drop liquid droplet on the gold electrode.

sensitivity of the QCM can be described as Gaussian function, like equation (9):

$$
S(r)=K e^{-\beta \frac{r^{2}}{r_{e}^{2}}}
$$

Where $K=1.6 \times 10^{12} \mathrm{~Hz} / \mathrm{Kg}$ is the largest sensitivity for QCM at the center of electrode, re is the radius of the gold electrode and $\beta$ is the constant.

In this study, the volume of sing-drop liquid was $0.5 \mu 1$ and The view of $0.5 \mu 1$ liquid droplet on the gold electrode surface was shown by Fig. (3).

In which, $\frac{\delta}{2}=\frac{1}{2} \sqrt{\frac{\eta}{\pi f_{0} \rho}}$ is the liquid decay length, and $\eta$ is viscosity for liquid, $\rho$ is the density for liquid, $f_{0}$ is fundamental resonant frequency for QCM. $r_{x}$ is the base radius for liquid droplet contain with decay length, rd is the base radius between liquid droplet to gold electrode.

The resonant frequency was shifted after liquid dropped on the surface of gold electrode, and it can be calculated as equation (10):

$$
\begin{aligned}
& \Delta f=\frac{2 \pi}{\pi r_{x}^{2}} \int_{0}^{r_{x}} k e^{-\beta \frac{r^{2}}{r_{e}^{2}}} \Delta m_{1} r d r \\
& +\frac{1}{\pi\left(r_{d}-r_{x}\right)^{2}} \int_{r_{x}}^{r_{d}} k e^{-\beta \frac{r^{2}}{r_{e}^{2}} \Delta m_{2} r d r}
\end{aligned}
$$

In which, $\Delta m_{1}=\rho \pi r_{x}^{2} \frac{\delta}{2}$ is the effective mass of the liquid droplet contain within decay length $\frac{\delta}{2}$ for base radius from center to $r_{x} ; \Delta m_{2}$ is the effective mass of the liquid droplet contain within decay length for base radius from $r_{x}$ to $\mathrm{r}_{\mathrm{d}}$. In this study, the decay length for water $\left(25^{\circ} \mathrm{C}\right)$ equal to $0.84 \times 10^{-4} \mathrm{~mm}$, liquid thickness is large compared to the decay length , then, the $\Delta m_{2}$ is much smaller than $\Delta m_{1}$, the influence of the $\Delta m_{2}$ could be negligent, equation(10) could be reduced as follow:

$\Delta f=\pi \delta \int_{0}^{r_{x}} k e^{-\beta \frac{r^{2}}{r_{e}^{2}}} \rho r d r$

Then:

$$
\Delta f=-\frac{1}{2} \pi \delta k \frac{r_{e}^{2}}{\beta} \rho\left[1-e^{-\beta \frac{r_{x}^{2}}{r_{e}^{2}}}\right]
$$

Consider $r_{x} \ll r_{e}$, equation (12) could be simplified to equation 13:

$$
\Delta f=-\frac{1}{2} \pi \delta k r_{x}^{2} \rho
$$

According to equation (13), the resonant frequency shift of QCM loaded by liquid droplet could be calculated.

\section{EXPERIMENTAL SECTION}

\subsection{Materials and Apparatus}

All the PZ crystals were AT-cut with a resonance frequency of $10 \mathrm{MHz}$. The crystal (diameter: $8 \mathrm{~mm}$, thickness: $0.185 \mathrm{~mm}$ ) was placed between two gold electrodes, mounted in metal holder with a plug. An symmetric electrode pattern was used so that the upper electrode and the lower electrode had same radius $(3 \mathrm{~mm})$. They were purchased from Tangshan JingYuan YuFeng Electronics co., Ltd (Hebei, China). The quartz crystal was driven at its resonant frequency with a homemade oscillator circuit and frequency measurement was performed with Agilent 531323a, with a precision of $0.1 \mathrm{~Hz}$ at a gate time of $0.1 \mathrm{~s}$. Micro-injector (range from $0.2 \mathrm{ul}$ to $10 \mathrm{ul}$ ) was obtained from KeXiao co., Ltd (Hang Zhou, China).

Phosphate-buffered saline (PBS) was composed of 137 $\mathrm{mM} \mathrm{NaOH}, 2.7 \mathrm{mM} \mathrm{KCL}, 8.0 \mathrm{mM} \mathrm{Na}_{2} \mathrm{HPO}_{4}$, and $1.5 \mathrm{mM}$ $\mathrm{KH}_{2} \mathrm{PO}_{4}(\mathrm{pH}$ 7.2).

\subsection{Measurements}

In order to guarantee that possible contaminants were removed, the QCM was dipped in $1.0 \mathrm{M} \mathrm{NaOH}$ for 10 min and in 1.0 M HCL for $10 \mathrm{~min}$ and then washed with distilled water at room temperature. After cleaning procedure, the crystal was air drying and the basic frequency $\left(\mathrm{F}_{0}\right)$ was taken. Then, the liquid dropped on the center of electrode surface using Micro-injector. In order to got the more information about the frequency response for liquid droplet, three different solutions include water, $0.01 \mathrm{~g} / \mathrm{ml} \mathrm{Nacl}, 0.1 \mathrm{~g} / \mathrm{ml} \mathrm{Nacl}$ were choose in this study. The different solution had different viscosity and different. All the experiments used same method and were conducted at same condition, include same temperature $\left(25^{\circ} \mathrm{C}\right)$, same humidity $(60)$ and same crystal 
Table 1. Result of frequency shift with same liquid volume $(0.5 \mu \mathrm{l})$.

\begin{tabular}{|c|c|c|c|}
\hline \multirow[t]{2}{*}{ Crystal Number } & \multicolumn{3}{|c|}{$\begin{array}{c}\Delta f(H z) \\
(0.5 \mu \mathrm{l})\end{array}$} \\
\hline & water & $0.01 \mathrm{~g} / \mathrm{mlNaCl}$ & $0.1 \mathrm{~g} / \mathrm{mlNaCl}$ \\
\hline 1 & 121 & 213 & 238 \\
\hline 2 & 132 & 184 & 225 \\
\hline 3 & 112 & 238 & 230 \\
\hline 4 & 138 & 167 & 221 \\
\hline 5 & 105 & 177 & 220 \\
\hline 6 & 108 & 176 & 223 \\
\hline average & 119 & 192 & 226 \\
\hline
\end{tabular}

Table 2. Comparison between measured data to calculated data.

\begin{tabular}{|c|c|c|}
\hline $\begin{array}{c}\text { Liquid } \\
(\mathbf{0 . 5} \boldsymbol{\mu l})\end{array}$ & $\begin{array}{c}\text { Measured Data } \\
\text { (average) }\end{array}$ & Calculated Data \\
\hline \hline Water & $119 \mathrm{~Hz}$ & $125 \mathrm{~Hz}$ \\
\hline $0.01 \mathrm{~g} / \mathrm{mlNaCl}$ & $192 \mathrm{~Hz}$ & $198 \mathrm{~Hz}$ \\
\hline $0.1 \mathrm{~g} / \mathrm{mlNaCl}$ & $226 \mathrm{~Hz}$ & $230 \mathrm{~Hz}$ \\
\hline
\end{tabular}

contours. Because of the symmetric electrode pattern, the device had high uniform mass sensitivity distribution [5-7].

\section{DATA AND ANALYSIS}

\subsection{Effect of the Viscosity}

Three different liquids include distill water, $0.01 \mathrm{~g} / \mathrm{ml}$ $\mathrm{NaCl}$ and $0.1 \mathrm{~g} / \mathrm{ml} \mathrm{NaCl}$ were used in this study. The volume of liquid used in this experimental were $0.5 \mu 1$ and All the liquid dropped on the center of the gold electrode. After liquid dropped, the resonant frequency $\left(F_{1}\right)$ measured by frequency counter (Agilent 531323a).

the resonant frequency shift were calculated by equation(14)

$\Delta f=F_{1}-F_{0}$

The experiments were repeated 6 times and the experimental results are summarized in (Table 1),

It is obviously to see from (Table 1) that the resonant frequency of all crystals decreased after liquid dropped on the center of gold electrode. It therefore founded that both the Sauerbrey and the Kanazawa equations may not be suitable for the interpretation of the frequency response of the QCM loaded by liquid droplet. The average frequency shift induced by $0.5 \mu 1$ water was $119 \mathrm{~Hz}$, induced by $0.01 \mathrm{~g} / \mathrm{mlNaCl}$ was $192 \mathrm{~Hz}$ and induced by $0.1 \mathrm{~g} / \mathrm{mlNaCl}$ was $205 \mathrm{~Hz}$. the experimental data indicated that the greater of the viscosity, the greater of the resonant frequency shift. In this study, the viscosity for water (25) was $0.8937 \mathrm{mpa} \cdot s$, and the viscosity for $0.1 \mathrm{~g} / \mathrm{mlNaCl}(25)$ was $1.039 \mathrm{mpa} \cdot s$, after drop the same volume on the gold electrode surface,the frequency shift induced by Nacl higher than water. Increased viscosity apparently increased the frequency shift. The resonant frequency shifted on account of the effective mass of liquid droplet contained within this decay length. The decay length $(\delta)$ depend on the viscosity $(\eta)$. If the viscosity and density of solution were increased, the decay length was increased, afterwards the resonant frequency decreased.

\subsection{Comparison Between Measured Data to Calculated Data}

In this study, three different liquid that had different viscosity and density were used for experimental. The resonant frequency shifted for different liquid could be calculated by equation (13). (Table 2) shows the frequency shift of the QCM as a liquid was dropped on the center of the gold electrode.

it is obviously to see from table3, all measured data had slight difference to calculated data. It can be deduced from above results: first, Sauerbrey and the Kanazawa equations may not suitable for liquid droplet on surface of QCM. Second, the viscosity and density would influence the resonant frequency. Third, the calculated data get from equation (13) agrees fairly will with the measured data.

\section{CONCLUSION}

The frequency response of QCM loaded by different liquid droplet was discussed in this study. Experiment data shows the relationship between liquid property include vis- 
cosity and density to resonant frequency, and the calculated data get from Theoretical formula which deduced in this paper agrees fairly will with the measured data.

\section{CONFLICT OF INTEREST}

The authors confirm that this article content has no conflict of interest.

\section{ACKNOWLEDGEMENTS}

This work was suported by the central college basic business expenses special funds of Southwest university for nationalities in 2013 (Young teachers fund projects), project number:13NZYQN06.
[2] K. Kanazawa, and J. G. Gordon, "The oscillation frequency of a quartz resonator in contact with liquid", Anal. Chim. Acta, vol.175, pp. $99-105,1985$

[3] A. C. Hillier, and M. D. Ward. "Scanning electrochemical mass sensitivity mapping of the quartz crystal microbalance in liquid media", Anal. Chem., vol. 64, pp. 2539-2554, 1992,

[4] H. F. Tiersten, "Linear Piezoelectric Plate Vibration", Plenum Press: New York, 1969, pp. 102-121

[5] F. Josse, and Y. Lee, "Analysis of the radial dependence of mass sensitivity for modified-electrode quartz crystal", Anal. Chem., vol. 70, pp. 237-247, 1998.

[6] D. M. Ward, and J. E. Delawski "Radial mass sensitivity of the quartz crystal microbalance in liquid media", Anal. Chem., vol. 63 , pp. 886-890, 1991.

[7] A. C. Hillier, and M. D. Ward. "Scanning electrochemical mass sensitivity mapping of the quartz crystal microbalance in liquid media", Anal. Chem., vol. 64, pp. 2539-2554, 1992.

\section{REFERENCES}

[1] G. Sauerbrey, "Use of quartz vibrator for weighting thin film on a microbalance", Z. Phys., vol.155, pp. 206-212, 1958.

Received: October 12, 2014

(C) Yang and Huang; Licensee Bentham Open.

This is an open access article licensed under the terms of the Creative Commons Attribution Non-Commercial License (http://creativecommons.org/licenses/by-nc/3.0/) which permits unrestricted, non-commercial use, distribution and reproduction in any medium, provided the work is properly cited. 